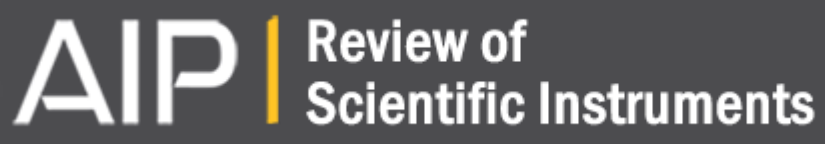

\section{Temperatures of Continuously Operated Mobile XRay Focal Spots}

\author{
Jesse W. M. DuMond, Bernard B. Watson, and Bruce Hicks
}

Citation: Review of Scientific Instruments 6, 183 (1935); doi: 10.1063/1.1751970

View online: http://dx.doi.org/10.1063/1.1751970

View Table of Contents: http://scitation.aip.org/content/aip/journal/rsi/6/7?ver=pdfcov

Published by the AIP Publishing

\section{Articles you may be interested in}

Magnetostatic focal spot correction for $\mathrm{x}$-ray tubes operating in strong magnetic fields using iterative optimization

Med. Phys. 39, 5567 (2012); 10.1118/1.4742060

SUC22004: Electrostatic Focal Spot Correction for XRay Tubes Operating in Strong Magnetic Fields Med. Phys. 38, 3380 (2011); 10.1118/1.3611510

Determination of xray tube focal spot position

Med. Phys. 20, 1029 (1993); 10.1118/1.597122

Alignment of xray tube focal spots for spectral measurement

Med. Phys. 9, 284 (1982); 10.1118/1.595086

New device for accurate measurement of the xray intensity distribution of xray tube focal spots Med. Phys. 2, 268 (1975); 10.1118/1.594190

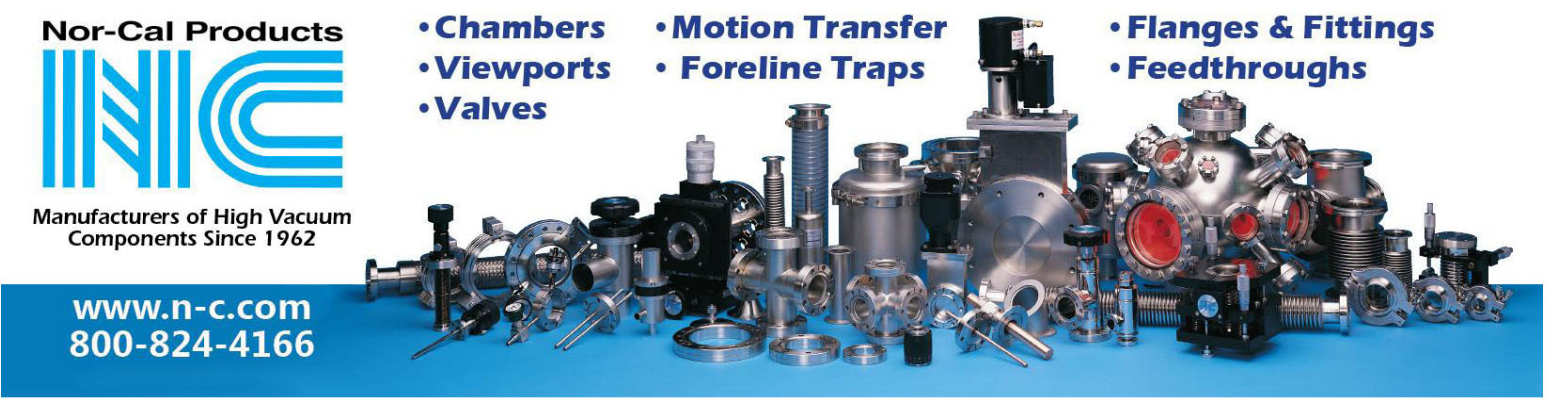




\section{CONTRIBUTED ARTICLES}

\section{Temperatures of Continuously Operated Mobile X-Ray Focal Spots}

Jesse W. M. DuMond, Bernard B. Watson and Bruce Hicks, California Institute of Technology, Pasadena, California

(Received May 2, 1935)

We use the adjective "mobile" to describe a focal spot in an x-ray tube which moves cyclically in a closed path relative to the target surface on which it is generated quite without reference to whether the spot be fixed or in motion with respect to the tube. Alex Müller and also A. Bouwers have made calculations of the thermal effects in such spots energized for very short fractions of one cycle of motion. This paper treats the case where the focal spot is energized continuously so that a "steady" state of thermal oscillation is set up. The solutions obtained refer only to conditions after this steady state of oscillation is reached. Only the ideal case of a spot of uniform intensity with sharp boundaries is treated. For simplicity the flow of heat from front target surface to cooling water is assumed one dimensional and normal to the front surface over an everywhere uniform thickness $d$. Curves are plotted showing the ratio of temperature rise in moving spots to that in fixed spots of the same size as a function of $r$ the size of the spot relative to the length of path it describes and of $\theta$ a variable depending on the speed of rotation, the thickness $d$ and the thermal constants of the target material. Curves of the ratio of permissible energy input for moving and fixed spots, respectively, as a function of the aforementioned variables are also shown. The paper gives a solution reduced to figures and curves of the heat flow equation for certain boundary conditions which to the author's knowledge has never before been obtained and therefore has some interest beyond its immediate application to $\mathrm{x}$-ray tubes. Certain mathematical difficulties of practical interest are also overcome in a way which may be helpful in other problems of similar nature. For the reader uninterested in mathematics the results are independently discussed in a separate section. Approximate methods are given for applying the results of the paper to targets consisting of two materials such as tungsten and copper.

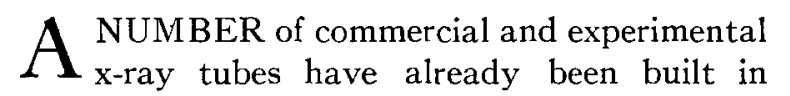
which either the focal spot moves over the face of a stationary target or more often the spot is stationary with respect to the tube but the target rotates or gyrates so that new regions of its surface are continually being presented under the focal spot. We use the adjective "mobile" in this paper to describe this motion of a focal spot relative to the target surface on which it is generated quite without reference to whether the spot be fixed or in motion with respect to the $\mathrm{x}$-ray tube. The mobile focal spot is a device resorted to for increasing the permissible power input without damage to the target. Two applications are to be distinguished according to whether the load be applied to the mobile focal spot for $a$ very brief time as in "snapshot" radiography of the organs of the human body for diagnostic work or continuously, that is to say, for a sufficiently long time to establish a dynamically steady cyclic thermal state in the path of the mobile focal spot. Theoretical calculations of the temperatures attained in mobile focal spots for briefly applied loads have been made by Alex. Müller ${ }^{1}$ and by A. Bouwers. ${ }^{2}$ To the author's knowledge no analysis has yet been made of the case of the continuously operated mobile $x$-ray focal spot and therefore this paper confines itself to this case.

\section{Statement of the Thermal Problem}

The mobile spot must execute a cyclic path on the surface of the target returning to any given position in its path after an interval of time $\tau$. To fix ideas we may suppose that the spot is a circular disk over which the energy input is uniform and of value, $w$, watts per unit area and this circular disk may be thought of as executing a circular path on the surface of the target as indicated in Fig. 1. If we single out

1 A. Müller, Proc. Roy. Soc. A125, 507 (1929).

2 A. Bouwers, Physica 10, 125 (1930). 


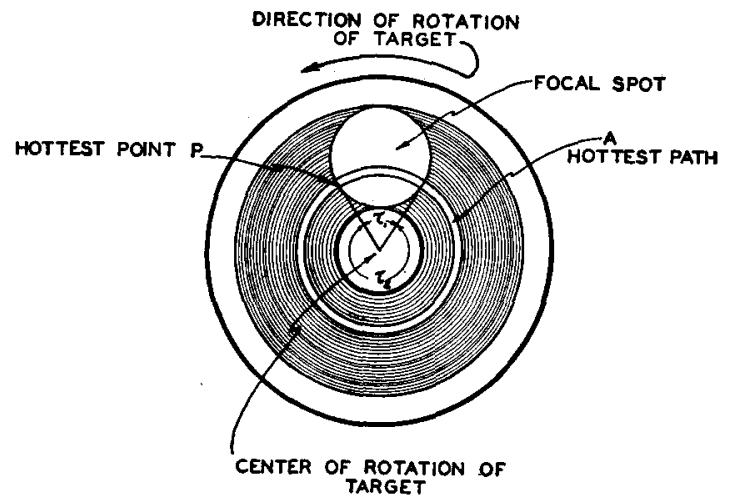

FIG. 1. As the target represented by the large circle rotates in the direction of the arrow the focal spot in the shape of a disk sweeps out the shaded circular path on the surface of the target. Elements of the target surface in the path $A$ spend the longest time under the focal spot and are hottest when they have reached the point $P$. The circular arcs marked $T_{1}$ and $T_{2}$ are proportional to the time of heating $T_{1}$ and the remainder of the cycle $T_{2}$, respectively. It is not of course necessary for the target to rotate and in a tube now under construction at this Institute it performs approximately a circular motion of translation imposed on it through an air tight sylphon joint. The stationary focal spot, since it has circular symmetry, therefore describes on the target surface a circular path just as here shown.

one small element of area on the target surface in the path of the focal spot this will receive heat at the surface rate, $w$, for $\tau_{1}$ seconds and will be left to itself for $\tau_{2}$ seconds where $\tau_{1}+\tau_{2}=\tau$ according to a square cycle like that indicated in Fig. 2. Evidently the highest temperature will invariably be reached at the surface and just at the end of each of the heating periods $\tau_{1}$. Also that element of target surface for which $\tau_{1}$ is of the longest duration will sustain the highest temperatures and this is therefore the only one we shall discuss.

We shall further assume for simplicity that our heat flow problem is one-dimensional, that is to say that the flow of heat is straight back normally from the target surface to the cooling water over a path $d \mathrm{~cm}$ in length without any lateral spreading of the heat. This will be a better approximation to the truth the larger the dimensions of the focal spot compared to the thickness, $d$, of the target. It seems reasonably clear that the error arising from this assumption will give results always on the conservative side from the point of view of design, that is to say, it should give the temperature rise too high rather than too low.

Of course no focal spot is uniformly loaded as

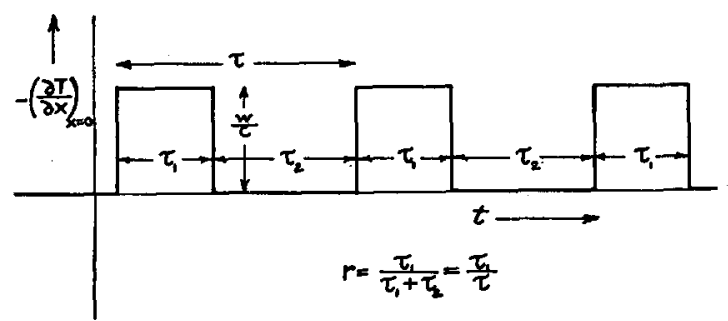

FIG. 2. Illustrating the "square cycle" of heat input as a function of time for any element of the target surface in the path $A$ of Fig. 1. The ordinates shown are actually the gradient of temperature at the target surface. This curve expresses the boundary condition for $x=0$.

we have assumed. Many of them are ring shaped having much lighter loading near the center of the disk. Local hot spots very frequently are present and may tend to become worse if they are images of local hot spots on the emitting cathode filament which is evaporating tungsten at these points at a faster rate and hence losing cross section in a localized way which continually exaggerates the trouble. All such things must be allowed for by a factor of safety applied on the basis of experience.

We shall content ourselves then with solving the following ideal problem. Heat is supplied to one end of a thermal conductor, an idealized cylinder or prism in the target with axis normal to the face of the target and extending back to the cooling water, $d \mathrm{~cm}$ long and having for convenience unit cross section. ${ }^{3}$ The conductor has a specific heat per unit mass $\sigma$, a mass density $\rho$, and a thermal conductivity $c . x$ is the distance measured from the heated end of the conductor to any point along its length, $t$ is the time and $T$ the temperature at any point in the conductor. The heat flow is purely longitudinal along $x$. One boundary condition is that when $x=d, T=T_{0}$ (the temperature of the cooling water). The other boundary condition when $x=0$ does not fix the temperature $T_{x=0}$ but rather refers to the temperature gradient $-(\partial T / \partial x)_{x=0}$ since it is the rate of energy input which is fixed at this point. Fig. 2 shows graphically the way in which the quantity $-(\partial T / \partial x)_{x=0}$ behaves as a function of time rising abruptly to

\footnotetext{
${ }^{3}$ We discuss in a section below how these ideal results may be adapted by approximation to the case of composite targets consisting of two metals such as a tungsten target imbedded in a copper backing.
} 
a value $-(\partial T / \partial x)_{x \triangleq 0}=w / c$ and remaining there for $\tau_{1}$ seconds, then falling abruptly to zero and remaining there for $\tau_{2}$ seconds and repeating this cycle indefinitely at the rate of $n$ cycles per second. For brevity let $r=\tau_{1} /\left(\tau_{1}+\tau_{2}\right)=\tau_{1} / \tau$. Evidently $0<r<1$. The maximum temperature $T_{\max }$ attained in the thermally steady cyclic state will obviously occur at the end of each heating period $\tau_{1}$ and at the point $x=0$. For convenience a complete nomenclature defining all symbols used in this article will be found at the end.

\section{Solution of the Thermal Problem}

The differential equation controlling the flow of heat along this ideal conductor is

$$
\partial^{2} T / \partial x^{2}=(\rho \sigma / c)(\partial T / \partial t) .
$$

Well-known particular solutions of this equation are

$$
\begin{aligned}
& T=T_{e}-g x, \\
& T=e^{-a x} \sin (2 \pi n t-a x) ; \\
& T=e^{-a x} \cos (2 \pi n t-a x) \\
& T=e^{-a x} e^{2(2 \pi n t-a x)}=e^{-(i+1) a x} e^{i 2 \pi n t} .
\end{aligned}
$$

or

The constant $a$ is immediately determined by substituting this solution into (1) and is

$$
a= \pm(\pi n \rho \sigma / c)^{\frac{1}{2}} \text {. }
$$

The most general solution for the state of steady thermal oscillation is a sum of the particular solutions multiplied by constant coefficients and is

$$
\begin{aligned}
T=T_{e}-g x+\sum_{s=1}^{\infty} A_{s} e^{-a_{s} x} \sin \left(2 \pi n_{s} t-a_{s} x\right) & +\sum_{s=1}^{\infty} B_{s} e^{-a_{s} x} \cos \left(2 \pi n_{s} t-a_{s} x\right) \\
& +\sum_{s=1}^{\infty} C_{s} e^{a_{s} x} \sin \left(2 \pi n_{s} t-a_{s} x\right)+\sum_{s=1}^{\infty} D_{s} e^{a_{s} x} \cos \left(2 \pi n_{s} t-a_{s} x\right) .
\end{aligned}
$$

In this solution $s$ takes only integer values from 1 to infinity. The appropriateness of this assumption is based on the fact that the boundary condition to be met at the front face of the target is a periodic function of the time and we know that the final steady state of thermal oscillation which interests us must also be a periodic function of the time. Our problem consists then in evaluating $A_{s}, B_{8}, C_{8}, D_{\varepsilon}$ and $g$ so as to fit the expression (2) to the following boundary conditions:

$$
\text { For } x=0-(\partial T / \partial x)_{x=0} c=\left\{\begin{array}{l}
w \text { for a period } \tau_{1} \\
0 \text { for a period } \tau_{2}
\end{array}\right\} \text { repeatedly as in Fig. } 2 .
$$

and for

$$
x=d \quad T=T_{0} \text {. }
$$

To satisfy the first boundary condition at $x=0$, we have

$$
-(\partial T / \partial x)_{x=0}=g+\sum_{s=1}^{\infty} a_{s}\left(A_{s}+B_{s}-C_{s}-D_{s}\right) \cos 2 \pi n_{s} t+\sum_{s=1}^{\infty} a_{s}\left(A_{s}-B_{s}-C_{s}+D_{s}\right) \sin 2 \pi n_{s} t .
$$

This then is the Fourier series which must be fitted to the periodic boundary condition of Fig. 2 . We have evidently $n_{s}=s n$. Evaluating the constant term and the Fourier coefficients we have

$$
g=w r / c,(4) \quad \begin{gathered}
A_{s}-B_{s}-C_{s}+D_{s}=\left(w / \pi c s a_{s}\right)(1-\cos 2 \pi s r), \\
A_{s}+B_{s}-C_{s}-D_{s}=\left(w / \pi c s a_{s}\right) \sin 2 \pi s r .
\end{gathered}
$$

Passing now to the second boundary condition at $x=d$ we have $T=T_{0}$ for all values of the time $t$. The expression (2) for $T$ is rearranged and set equal to $T_{0}$ while $x$ is set equal to $d$. (In this the value (4) determined for $g$ is inserted.) 


$$
\begin{aligned}
T=T_{0}=T_{e}-w r d / c+ & +\sum_{s=1}^{\infty}\left(A_{s} e^{-a_{s} d} \cos a_{s} d+B_{s} e^{-a_{s} d} \sin a_{s} d+C_{s} e^{a_{s} d} \cos a_{s} d-D_{s} e^{a_{s} d} \sin a_{s} d\right) \sin 2 \pi n_{s} t \\
& +\sum_{s=1}^{\infty}\left(-A_{s} e^{-a_{s} d} \sin a_{s} d+B_{s} e^{-a_{s} d} \cos a_{s} d+C_{s} e^{a_{s} d} \sin a_{s} d+D_{s} e^{a_{s} d} \cos a_{s} d\right) \cos 2 \pi n_{s} t .
\end{aligned}
$$

This Fourier series in $t$ can be equal to the constant $T_{0}$ for all values of $t$ only if all the coefficients of $\sin 2 \pi n_{s} t$ and $\cos 2 \pi n_{s} t$ vanish. The two summations in the above expression are thus zero which determines $T_{e}$

$$
T_{e}=T_{0}+w r d / c
$$

At the same time the vanishing of the coefficients of $\sin 2 \pi n_{s} t$ and $\cos 2 \pi n_{s} t$ yield the two following conditions

$$
\begin{gathered}
A_{s} e^{-a_{s} d} \cos a_{s} d+B_{s} e^{-a_{s} d} \sin a_{s} d+C_{s} e^{a_{s} d} \cos a_{s} d-D_{s} e^{a_{s} d} \sin a_{s} d=0, \\
-A_{s} e^{-a_{s} d} \sin a_{s} d+B_{s} e^{-a_{s} d} \cos a_{s} d+C_{s} e^{a_{s} d} \sin a_{s} d+D_{s} e^{a_{s} d} \cos a_{s} d=0
\end{gathered}
$$

Now Eqs. (5), (6), (8) and (9) regarded as four simultaneous equations are sufficient to solve for the four constants $A_{s}, B_{s}, C_{s}$ and $D_{s}$, and we thus obtain

$$
\begin{aligned}
& A_{s}+C_{s}=\frac{P_{s} \sinh a_{s} d \cosh a_{s} d+Q_{s} \sin a_{s} d \cos a_{s} d}{\cos ^{2} a_{8} d+\sinh ^{2} a_{s} d}, \\
& D_{s}+B_{s}=\frac{P_{s} \sin a_{s} d \cos a_{s} d-Q_{s} \sinh a_{s} d \cosh a_{s} d}{\cos ^{2} a_{s} d+\sinh ^{2} a_{s} d} .
\end{aligned}
$$

Where $P_{s}$ and $Q_{s}$ are abbreviations for the expressions

$$
\begin{aligned}
& A_{s}-C_{s}=\left(w / 2 \pi c s a_{s}\right)(1-\cos 2 \pi s r+\sin 2 \pi s r)=P_{s}, \\
& D_{s}-B_{s}=\left(w / 2 \pi c s a_{s}\right)(1-\cos 2 \pi s r-\sin 2 \pi s r)=Q_{s} .
\end{aligned}
$$

If we refer now to our original solution (2) the maximum temperature which we will designate by $T_{\max }$ will occur for $x=0$ and at the end of each period of heat supply that is for $\sin 2 \pi n_{s} t=\sin 2 \pi s r$ and $\cos 2 \pi n_{s} t=\cos 2 \pi s r$. We place then $x=0$ in (2), substitute the value ( 7 ) for $T_{e}$, insert the values (10), (11), (12) and (13) into the resulting expression as well as the conditions $\sin 2 \pi n_{s} t=\sin 2 \pi s r$, $\cos 2 \pi n_{s} t=\cos 2 \pi s r$ which pick the hottest instant in the thermal cycle and obtain $T_{\max }$

$$
T_{\max }=T_{0}+\underset{c}{w} d+\frac{w}{2 \pi c} \sum_{s=1}^{\infty} \frac{1}{s a_{s}} \frac{p_{s} \sinh a_{s} d \cosh a_{s} d-q_{s} \sin a_{s} d \cos a_{s} d}{\cos ^{2} a_{s} d+\sinh ^{2} a_{s} d},
$$

where

$$
p_{s}=1+\sin 2 \pi s r-\cos 2 \pi s r,
$$

$$
q_{s}=1-\sin 2 \pi s r-\cos 2 \pi s r
$$

$$
a_{s}=(\pi \rho \sigma / c)^{\frac{1}{2}}(s n)^{\frac{1}{2}}
$$

This expression can be somewhat simplified for purposes of computation as follows:

Let $a_{s} d=\theta_{s} ; d(\pi \rho \sigma / c)^{\frac{1}{2}} n^{\frac{1}{2}}=\theta$; then $\theta_{s}=\theta s^{\frac{1}{2}}$. The summation thus becomes a function of only two dimensionless variables $\theta$ and $r$ (the latter involved in $p_{s}$ and $q_{s}$ ). By use of the relations

$\sinh \theta_{s} \cosh \theta_{s}=\frac{1}{2} \sinh 2 \theta_{s}, \quad \sin \theta_{s} \cos \theta_{s}=\frac{1}{2} \sin 2 \theta_{s}, \quad \cos ^{2} \theta_{s}+\sinh ^{2} \theta_{s}=\frac{1}{2} \cosh 2 \theta_{s}+\frac{1}{2} \cos 2 \theta_{s}$ we obtain :

$$
T_{\max }=T_{0}+\underset{c}{w}-r d+\frac{w d}{\pi c} \sum_{s=1}^{\infty} \frac{1}{s \theta_{s}{ }^{\prime}} \frac{p_{s} \sinh \theta_{s}{ }^{\prime}-q_{s} \sin \theta_{s}{ }^{\prime}}{\cosh \theta_{s}{ }^{\prime}+\cos \theta_{s}{ }^{\prime}} ; \quad \text { where } \theta_{s}{ }^{\prime}=2 \theta_{s}
$$


For convenience in plotting we define the dimensionless ratio $R=\left(T_{\max }-T_{0}\right) /\left(T_{s}-T_{0}\right)$, where $T_{s}$ is the temperature attained at the surface of the focal spot if it is stationary. $T_{s}-T_{0}=w d / c$. Thus $R$ is the ratio of maximum temperature rises attained in moving and stationary focal spots, respectively, and its reciprocal $1 / R$ represents how many times as much energy input can be safely tolerated in the moving spot than would be safe in the stationary spot.

$$
1 / R=W_{m} / W_{s}, \quad R=r+\frac{1}{\pi} \sum_{s=1}^{\infty} \frac{1}{s \theta_{s}{ }^{\prime}} \frac{p_{s} \sinh \theta_{s}{ }^{\prime}-q_{s} \sin \theta_{s}{ }^{\prime}}{\cosh \theta_{s}{ }^{\prime}+\cos \theta_{s}{ }^{\prime}} .
$$

Now let:

$$
F_{+}\left(\theta_{s}{ }^{\prime}\right)=\left(\sinh \theta_{s}{ }^{\prime}+\sin \theta_{s}{ }^{\prime}\right) /\left(\cosh \theta_{s}{ }^{\prime}+\cos \theta_{s}{ }^{\prime}\right)
$$

and

$$
F_{-}\left(\theta_{s}{ }^{\prime}\right)=\left(\sinh \theta_{s}{ }^{\prime}-\sin \theta_{s}{ }^{\prime}\right) /\left(\cosh \theta_{s}{ }^{\prime}+\cos \theta_{s}{ }^{\prime}\right) \text {. }
$$

We can now insert the values of $p_{s}$ and $q_{s}(15)$ and (16)

$$
R=r+\frac{1}{\pi} \sum_{s=1}^{\infty} \frac{1}{s \theta_{s}{ }^{\prime}} F_{-}\left(\theta_{s}{ }^{\prime}\right)+\frac{1}{\pi} \sum_{s=1}^{\infty} \frac{1}{s \theta_{s}{ }^{\prime}} F_{+}\left(\theta_{s}{ }^{\prime}\right) \sin 2 \pi s r-\frac{1}{\pi} \sum_{s=1}^{\infty} \frac{1}{s \theta_{s}{ }^{\prime}} F_{-}\left(\theta_{s}{ }^{\prime}\right) \cos 2 \pi s r .
$$

In Fig. 3 we plot $F_{-}\left(\theta_{s}{ }^{\prime}\right)$ and $F_{+}\left(\theta_{s}{ }^{\prime}\right)$ against $\theta_{s}{ }^{\prime}$. It is evident that as $s$ and $\theta_{s}{ }^{\prime}=2 \theta s^{\frac{1}{3}}$ increase in passing to higher and higher terms of the summations (21) the functions $F_{-}$and $F_{+}$both converge rapidly to unity so that by the time $\theta_{s}{ }^{\prime}=2 \pi$ they each differ from unity by less than four parts in one thousand and never differ by more than this thereafter. Thus if we set $F_{+}$and $F_{-}$equal to unity for all summation terms beyond and including the lowest one for which $\theta_{s} \geqslant \pi$ the relative error in this residue of the series cannot exceed four parts in one thousand. Thus the form we have used for numerical calculation in which $\alpha$ is the lowest positive integer greater than $\pi^{2} / \theta^{2}$ becomes:

$$
\begin{aligned}
& R=r+\frac{1}{\pi} \sum_{s=1}^{\alpha-1} \frac{1}{s \theta_{s}{ }^{\prime}} F_{-}\left(\theta_{s}{ }^{\prime}\right)+\frac{1}{\pi} \sum_{s=1}^{\alpha-1} \frac{1}{s \theta_{s}{ }^{\prime}} F_{+}\left(\theta_{s}{ }^{\prime}\right) \sin 2 \pi s r-\frac{1}{\pi} \sum_{s=1}^{\alpha-1} \frac{1}{s \theta_{s}{ }^{\prime}} F_{-}\left(\theta_{s}{ }^{\prime}\right) \cos 2 \pi s r \\
& +\frac{1}{\pi} \sum_{s=\alpha}^{\infty} \frac{1}{s \theta_{s}{ }^{\prime}}+\frac{1}{\pi} \sum_{s=\alpha}^{\infty} \frac{1}{s \theta_{s}{ }^{\prime}} \sin 2 \pi s r-\frac{1}{\pi} \sum_{s=\alpha}^{\infty} \frac{1}{s \theta_{s}{ }^{\prime}} \cos 2 \pi s r .
\end{aligned}
$$

Or briefly

$$
R=r+(1 / \pi)\left(x+y-z+x^{\prime}+y^{\prime}-z^{\prime}\right)=r+(1 / \pi) X(\theta, r) \text {. }
$$

The values of $r$ and $\theta$ for which $x, y, z, x^{\prime}, y^{\prime}, z^{\prime}$ were computed together with values of $\alpha$ where the finite sum was broken off are

\begin{tabular}{c|ccccccc}
$r=$ & $1 / 12$, & $1 / 6$, & $1 / 3$, & $1 / 2$, & $2 / 3$, & $5 / 6$, & $11 / 12$ \\
\hline $\begin{array}{c}\theta=0.5 \\
\alpha=40\end{array}$ & 1.0 & 1.5 & 2 & 3 & 4 & 5 & \\
& 10 & 5 & 3 & 2 & 1 & 1 &
\end{tabular}

For the finite summations $x, y$ and $z$ the values of $F_{-}$and $F_{+}$. were picked off the plotted curve Fig. 3 for the appropriate values of $\theta_{s}{ }^{\prime}=\theta^{\prime} s^{\frac{1}{2}}$ corresponding to the different integer values of $s$ (a different set for each value of $\theta^{\prime}$ ) with a great saving of labor in computation.

The infinite sum $x^{\prime}$ requires the evaluation of

$$
\sum_{s=\alpha}^{\infty} s^{-\frac{3}{2}} \equiv \sum_{s=1}^{\infty} s^{-\frac{3}{2}}-\sum_{s=1}^{\alpha-1} s^{-\frac{1}{1}}
$$

The finite sum here was evaluated by plain addition. The very slowly converging infinite sum $\sum_{s=1}^{\infty} s^{-\frac{3}{2}}$ was found to have the value 2.6124 . This was obtained by summing a number of terms $\psi$ and calculating the residue by means of the Euler-McLaurin integral summation formula. ${ }^{4}$ This latter, as is well known, is an infinite series in which the Bernoulli numbers appear and which eventually diverges. Nevertheless the error committed by breaking it off at any given term can in this case be placed between limits by means of a very simple formula and these limits may be made as small as desired if $\psi$ in the series to be summed be chosen suffi-

\footnotetext{
'See for example Steffenson's book, Interpolation, Williams and Wilkins, p. 133.
} 
ciently large. We took $\psi$ equal to 20 terms and the error in evaluating the residue after 20 terms was in the sixth decimal place only of the final value of $\sum_{s=1}^{\infty} s^{-1}$.

We are much indebted to Dr. Morgan Ward ${ }^{5}$ of this Institute who devised a most ingenious and beautiful method not only for summing the slowly converging sums $y^{\prime}$ and $z^{\prime}$ but for any series of the form

$$
\sum_{n=a+1}^{\infty} \phi(n) e^{2 n \pi i x}, \quad 0<x<1 .
$$

The method requires only the construction of a small table of the differences of $\phi(n)$ of different orders.

\section{Discussion of Result and Its Exhibition in Graphical Form}

For a uniformly loaded focal spot the ratio

$$
\frac{\text { Maximum temperature rise of moving spot }}{\text { Temperature rise of stationary spot }}=R
$$

is shown to be given by Eq. (29) as

$$
R=r+(1 / \pi) X(\theta, r),
$$

where $\theta=d n^{\frac{1}{2}}(\pi \rho \sigma / c)^{\frac{1}{2}}, r=\tau_{1} /\left(\tau_{1}+\tau_{2}\right)$ (see Fig. 1). See Nomenclature at end of article for significance of symbols. In Fig. 4 we show $R$ plotted against $r$ for various values of $\theta$ while in Fig. 5 $R$ is plotted against $\theta$ for various values of $r$. Fig. 6 which is simply the reciprocal of $R$ plotted against $\theta^{2}$ gives the ratio

$$
\frac{\text { Permissible watts input for moving spot }}{\text { Permissible watts input for stationary spot }}=\frac{W_{m}}{W_{s}}
$$

as a function of $\theta^{2}$, an abscissa directly proportional to the speed of rotation $n . \theta^{2}=\pi d^{2}(\rho \sigma / c) n$. Thus the multiplication of permissible power in virtue of rotation can be read off of this chart as a function of the speed if the constant $\pi d^{2}(\rho \sigma / c)$ is first evaluated for the target in question. As an example we have attached a scale in this figure where the speed appears directly for a copper target of thickness $d=0.5 \mathrm{~cm}$. For such a target nine times as much power can be safely applied at 20 r.p.s. as could be safely applied for a stationary spot when the spot diameter divided

\footnotetext{
${ }^{6}$ Morgan Ward, Am. Math. Monthly 41, 563 (1934).
}

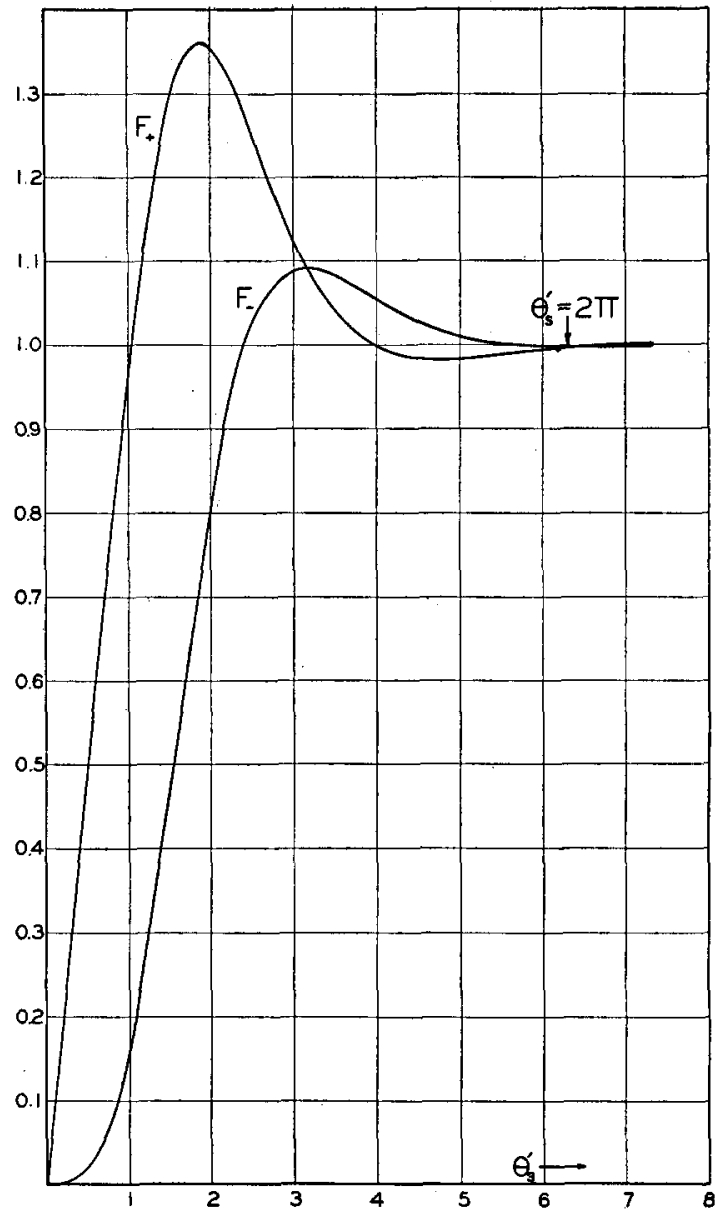

FIG. 3. Illustrating the behavior of the functions $F_{+}$and $F_{-}$. The successive maximum and minimum values of each of these functions differ from unity by very rapidly diminishing amounts as can readily be verified by differentiating their analytical expressions.

by the circumference swept out is $r=.03$. These curves approach in each case an asymptotic value $1 / r$ for $\theta^{2}=\infty$ that is for infinite speed of rotation. This is to be expected since at infinite speed the surface temperature will be uniform over the entire ring swept out by the focal spot at its widest point (the ring $A$ in Fig. 1) and the area of this ring is $1 / r$ times the corresponding area for the stationary spot. Thus $1 / r=W_{m} / W_{s}$ for infinite speed and no finite speed of rotation can obviously give as large a multiplication of permissible power input. A qualitative idea of the effectiveness of rotation in smoothing out local hot spots in the focal spot can be gained by noting in Fig. 6 how large the multiplication of permissible power input becomes for small 


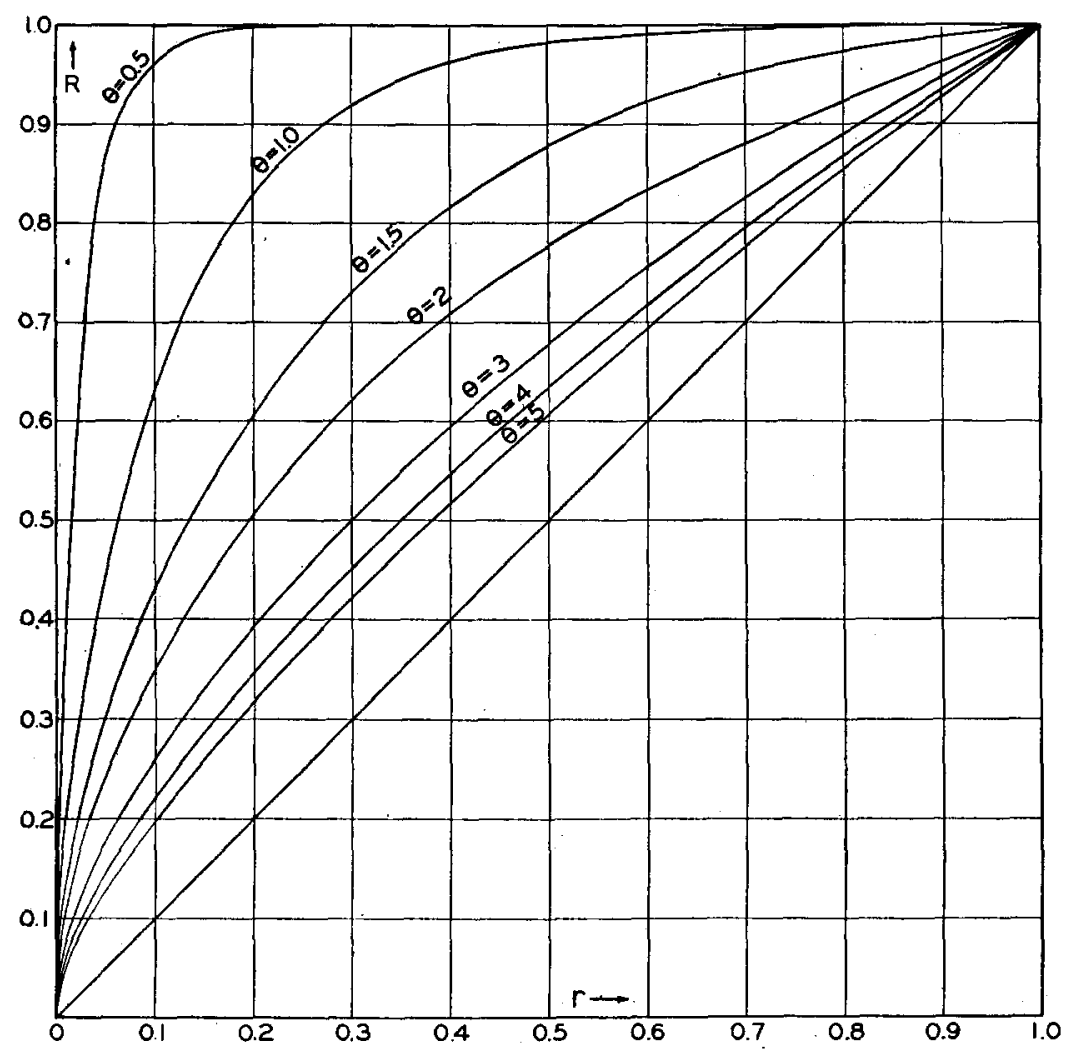

FIG. 4. The ordinates $R$ show the ratio of the maximum target surface temperature attained in a mobile focal spot to the temperature of the same spot when stationary. $\theta=d(\pi \rho \sigma / c)^{\frac{1}{2}} n^{\frac{1}{2}}$ becomes infinite for infinite speeds of rotation and the curves merge with the $45^{\circ}$ straight line. At very high speeds then these curves show. that the maximum surface temperature for the mobile spot will approach simply $r$ times that for the stationary spot as one would expect since $r$ is the fraction of the time the heat is applied to any surface element. Lower temperatures than this cannot be attained by rotation. The excess of temperature above the $45^{\circ}$ straight line for lower speeds thus represents the effect of imperfect "ironing out" of the temperature waves at the surface by thermal storage.

diameter focal spots, that is to say small $r$, even at very moderate speeds (e.g., at 6 r.p.s. if $r=0.01$ the multiplication is nearly 10$)$. Since the abscissa of the curve in Fig. 6 is $\theta^{2}=\pi d^{2}(\rho \sigma / c) n$ a large increase in multiplication of permissible power input results by simply increasing $d$, but the permissible power input cannot itself be increased by increasing $d$. Quite the contrary is the case because the permissible power input for the stationary case $W_{s}$ is itself inversely proportional to $d$ and the increase in multiplication factor $W_{m} / W_{s}$ with increasing $d$ is insufficient to make up for this. The permissible power input for the stationary spot is on our assumption of no lateral spreading of the heat

$$
W_{8}=c T^{*} A / d,
$$

where $T^{*}$ is the surface temperature for safe operation of the target material, $A$ is the area of the spot and $c$ and $d$ have the values defined above.

In the curves of Fig. 4 which represent $R$ the ratio of temperatures of moving spot and stationary spot the diagonal $45^{\circ}$ straight line is the curve for $\theta=\infty$ or infinite speed of rotation. If the ordinates of Fig. 4 are multiplied by $w d / c$ they give the actual temperature rises attained as set out in the expression (14.1). The $45^{\circ}$ straight line then corresponds to the term wrd/c in that formula. If we let $\theta^{\prime}=\infty$ in the summation term of (14.1) the summand evidently vanishes for all values of $s$, and only the term wrd/c remains in the temperature rise above $T$. Again we point out, however, that if $\theta^{\prime} \rightarrow \infty$ on account 


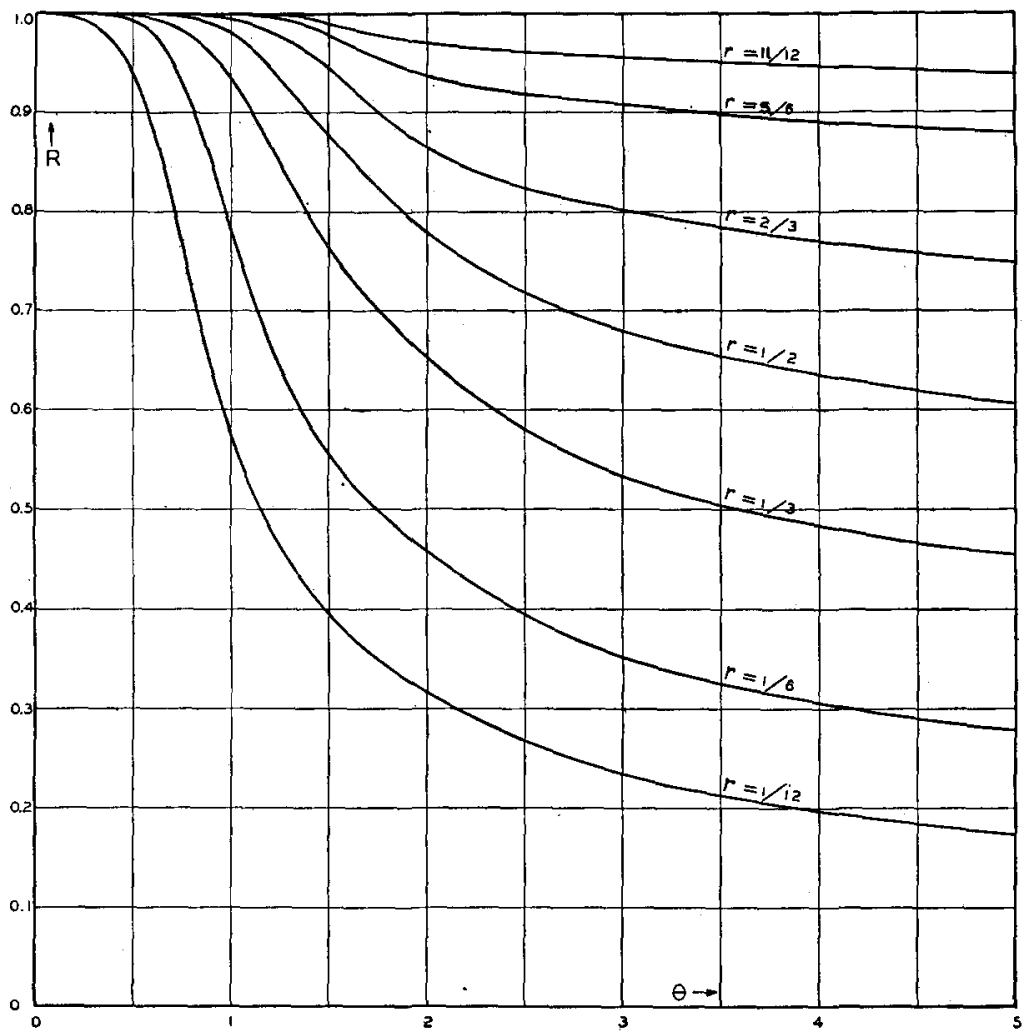

FIG. 5. The same quantity $R$ as in Fig. 4 is plotted here against $\theta$ for various values of $r$. $R$ has been plotted in these two different ways to facilitate interpolation for values of $\theta$ and $r$ between the contours.

of an unlimited increase in $d$ the coefficient of the summation term, which contains $d$ in the numerator will prevent the term as a whole from vanishing. We see thus that the rise above the $45^{\circ}$ line in Fig. 4 represents the summation term of (14.1) which we have been at so much pains to deduce and compute and which represents the additional rise in maximum temperature for a moving spot of finite speed above the temperature for infinite speed which is the lowest altainable.

It is of some mathematical interest to show that for any finite value of $r$ where $\theta$ vanishes (zero speed) the temperature rise given by (14.1) actually approaches $w d / c$, the temperature rise for a stationary focal spot. We are able to show that when $r$ is finite and $\theta \rightarrow 0$ the expression

$$
R=r+\frac{1}{\pi} \sum_{s=1}^{\infty} \frac{1}{s \theta_{s}{ }^{\prime}} \frac{p_{s} \sinh \theta_{s}{ }^{\prime}-q_{s} \sin \theta_{s}{ }^{\prime}}{\cosh \theta_{s}{ }^{\prime}+\cos \theta_{s}{ }^{\prime}}=r+\frac{1}{\pi} X
$$

approaches unity.
The summation $X$ regarded as a function of $\theta^{\prime}$ is convergent for all values of $\theta^{\prime}$ in the range zero to infinity but it is not uniformly convergent over this range. As $\theta^{\prime}$ diminishes, $X$ converges more and more slowly. Thus if we take the sum of this series to any fixed limiting number of terms, $e$, the vanishing quantity $\theta^{\prime}$ can become sufficiently small before it reaches zero so that the residue of the series after the $e$ th term will be finite and cannot be neglected no matter how large the value at which $e$ was fixed. Though we cannot neglect this residue summation, we can replace it with an integral which can be made to differ from the residue as little as we please by taking $e$ sufficiently large. Unfortunately space limitations do not permit us to reproduce our proof here but the result is that for $\theta \rightarrow 0, R \rightarrow 1$. For $\theta=0$ exactly it is easy to show that $R=1 / 2$, an excellent example of the Gibbs phenomenon which is sketched in Fig. 7. 


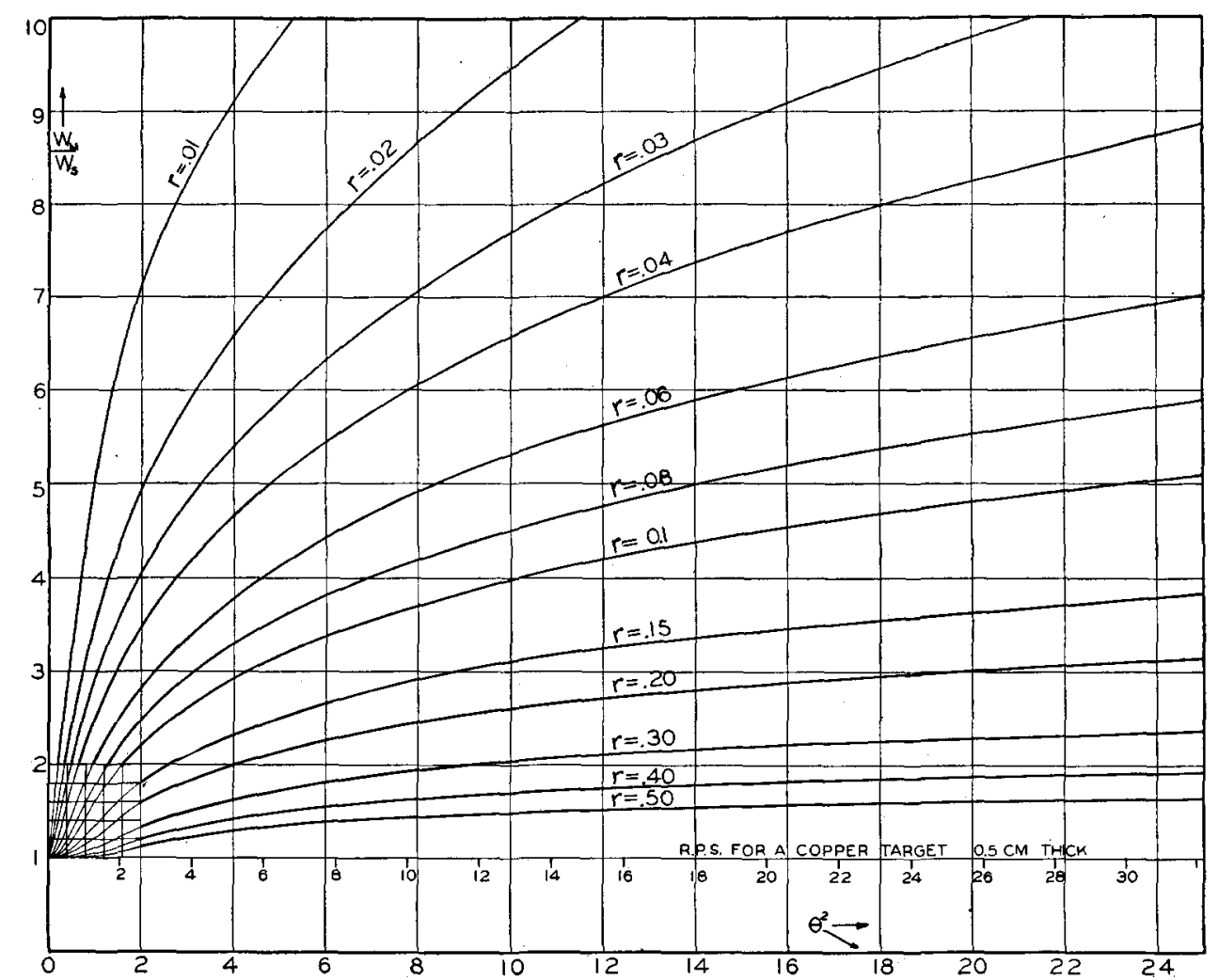

FIG. 6. The results of this paper are best summarized on this chart which shows the multiplication of permissible power by virtue of rotation for different speeds $\left(\theta^{2}=\left(\pi d^{2} \rho \sigma / c\right) \times\right.$ r.p.s. $)$ and for different sizes of focal spot relative to path swept out $(r)$.

Application to Composite Targets Such as Tungsten AND COPPER

This paper makes no effort to cover rigorously the much more complicated case of a target consisting of two materials of very different thermal constants such as tungsten inlaid into a copper backing. Since this case is of such great practical interest however we shall discuss approximate ways of adapting our present results for application to such targets.

Let $d_{1}$ be the thickness of the material to which the heat is applied (the tungsten) and $d_{2}$ the thickness of the backing material. The thermal quantities, $\rho_{1} \sigma_{1} / c_{1}$ and $\rho_{2} \sigma_{2} / c_{2}$ apply, respectively, to the two materials. For copper $\rho \sigma / c$ equals about 1 and for tungsten about 2. The choice of one appropriate way of approximating this new situation turns on the question as to how important the amplitude of the fluctuations of temperature with time are at the interface between the two materials in comparison to $T_{\max }-T_{0}$. In the absence of an exact theory this can only be decided by good judgment. The amplitude of the temperature oscillations at the interface will evidently be the less important the greater $d_{1} / c_{1}$ in comparison to $d_{2} / c_{2}$ and the greater $d_{1}\left(\rho_{1} \sigma_{1} / c_{1}\right)^{\frac{3}{2}}$ in comparison to $d_{2}\left(\rho_{2} \sigma_{2} / c_{2}\right)^{\frac{1}{2}}$. A further criterion can be obtained by noting that the principal components of these oscillations as seen from Eq. (2) suffer a damping such that their amplitudes decrease to but little more than one-third the initial value $(1 / e$ to be precise $)$ in a distance $(c /(\pi n \rho \sigma))^{\frac{1}{2}}$ which is slightly less than one millimeter for tungsten if $n=20$ r.p.s. The "ironing out" of the heat waves is of course here regarded not as the effect of lateral flow of heat which we have excluded but the effect of thermal storage in the material traversed.

Suppose then that we can regard uniform heat flow as substantially established by the time the heat has reached the copper backing. We can then apply formula (14.1) to the tungsten alone: 


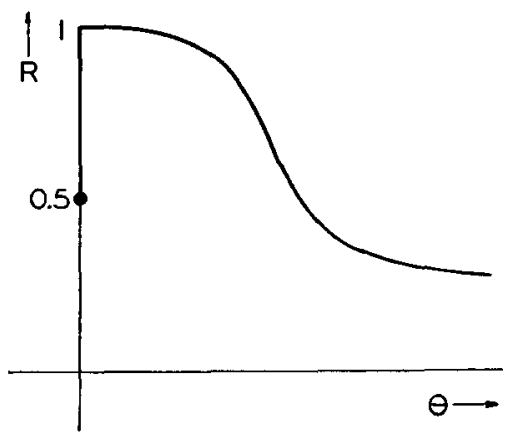

FIG. 7. Illustrating the behavior of $R$ for finite values of $r$ as $\theta$ tends toward zero. At the point $\theta=0$ the function executes a sudden jump from the value 1 to the value $1 / r$, an example of the Gibbs phenomenon.

$$
T_{\max }-T_{\text {interface }}=w r d_{1} / c_{1}+\left(w d_{1} / \pi c_{1}\right) X\left(\theta_{1}, r\right) .
$$

Also $T_{\text {interface }}-T_{0}=w r d_{2} / c_{2}$ because the watts per unit area have in the copper been reduced to the value $r w$ instead of $w$. Hence

$$
T_{\max }-T_{0}=w r d_{1} / c_{1}+w r d_{2} / c_{2}+\left(w d_{1} / \pi c_{1}\right) X\left(\theta_{1}, r\right) .
$$

For a stationary focal spot the temperature rise is

$$
T_{8}-T_{0}=w\left(d_{1} / c_{1}+d_{2} / c_{2}\right) .
$$

We have then the new formula for $R_{c}$ for the composite target

$$
\frac{T_{\max }-T_{0}}{T_{s}-T_{0}}=R_{c}=r+\frac{1}{\left(1+c_{1} d_{2} / c_{2} d_{1}\right)}-\frac{1}{\pi}-X\left(\theta_{1}, r\right) .
$$

Thus if we compute the value of

$$
\theta_{1}=d_{1}\left(\pi \rho_{1} \sigma_{1} / c_{1}\right)^{\frac{1}{2}} n^{\frac{1}{2}}
$$

for the tungsten alone we can pick out a value $R_{1}$ on the curves of say Fig. 4 or Fig. 5 at the point corresponding to $\theta_{1}$ and $r$. Since this $R_{1}$ is

$$
R_{1}=r+(1 / \pi) X\left(\theta_{1}, r\right)
$$

the desired quantity $R_{\mathrm{c}}$ can be obtained from $R_{1}$ by means of the formula

$$
R_{c}=\frac{R_{1}-r}{1+c_{1} d_{2} / c_{2} d_{1}}+r .
$$

The approximate multiplication of permissible power is simply $1 / R_{c}$ as before.

Since there is considerable uncertainty involved in the assumption that the heat waves have been completely "ironed out" before reaching the copper and since if these temperature fluctuations are present and not negligible at the interface the above method does not err on the conservative side it is best to allow some margin of safety when the above method is used.

On the other hand a perfectly safe assumption but one which may be a little too pessimistic is to assume the entire thickness $d_{1}+d_{2}$ of the composite target to have the thermal constants of the tungsten. The value of $R$ computed in this way will certainly be an upper limit to the actual case and the truth will lie somewhere between the limits computed in these last two ways. An alternative way of obtaining a value of $R$ for composite targets which is sure to be too large (i.e., on the conservative side in design) is to assume that the whole energy input per unit area $w$ is applied to the copper at its interface with the tungsten and compute $T_{\max }-T_{0}$ for this interface and then add to this a temperature rise through the tungsten as though the target were not rotating (i.e., for steady flow). This will be nearer the truth than the last conservative method if the tungsten is very thin compared to the copper, whereas the former will be better if the reverse is the case. Probably $R$ should be computed by both of these conservative methods for the particular case of interest and the lower of the two chosen as the upper limit for comparison with the lower limit $R_{c}$ whose method of computation was described first. It is very easy to show that the last method of approximating $R_{c}$ gives

$$
R_{c}=\frac{1}{1+c_{1} d_{2} / c_{2} d_{1}}+\frac{1}{1+c_{2} d_{1} / c_{1} d_{2}}\left\{r+\frac{1}{\pi} X\left(\theta_{2}, r\right)\right\},
$$

where $\theta_{2}=d_{2}\left(\pi \rho_{2} \sigma_{2} / c_{2}\right)^{\frac{1}{2}} n^{\frac{1}{3}}$. Calling the expression in curly brackets $R_{\mathbf{2}}$ in harmony with our previous nomenclature we can sum up our three approximate formulae for $R_{c}$ which we will call in the order they have been discussed $R_{c}{ }^{\mathrm{I}}, R_{c}{ }^{\mathrm{II}}$ and $R_{c}^{\text {III }}$, respectively, as follows :

$$
\begin{aligned}
& R_{c}{ }^{\mathrm{I}}=r+\frac{R_{1}-r}{1+c_{1} d_{2} / c_{2} d_{1}} \leqq \text { True } R_{c} ; \\
& R_{1} \text { obtained from curves using } \\
& \theta_{1}=d_{1}\left(\pi \rho_{1} \sigma_{1} / c_{1}\right)^{\frac{1}{2}} n^{\frac{1}{2}},
\end{aligned}
$$


$R_{c}{ }^{\mathrm{II}} \geqq$ True $R_{c} ; R_{c}{ }^{\mathrm{II}}$ obtained from curves using

$$
\theta=\left(d_{1}+d_{2}\right)\left(\pi \rho_{1} \sigma_{1} / c_{1}\right)^{\frac{1}{2}} n^{\frac{1}{3}}
$$

$$
R_{c}^{\mathrm{III}}=\frac{1}{1+c_{1} d_{2} / c_{2} d_{1}}+\frac{R_{2}}{1+c_{2} d_{1} / c_{1} d_{2}} \geqq \text { True } R_{c} ;
$$

$R_{2}$ obtained from curves using

$$
\theta_{2}=d_{2}\left(\pi \rho_{2} \sigma_{2} / c_{2}\right)^{\frac{1}{2}} n^{\frac{1}{2}} \text {. }
$$

The true value of $R_{c}$ will lie above $R_{c}{ }^{\mathrm{I}}$ and below either $R_{c}{ }^{\text {II }}$ or $R_{c}{ }^{\text {III }}$ whichever is the lower. The method should place $R$ between fairly close limits in most cases.

\section{ACKNOWLEDGMENTS}

In conclusion we wish to express our appreciation to Dr. Morgan Ward, Dr. W. V. Houston and Dr. H. Bateman, all three of this Institute, for their very helpful advice in the solution of this problem and to Mr. Paul Youtz for assistance in the numerical computations. The construction at this Institute of a gyrating target $\mathrm{x}$-ray tube whose design occasioned this mathematical investigation was made possible along with the constant potential generating equipment large enough to supply it with $30 \mathrm{kw}$ continuously through the generosity of Dr. Leon L. Watters of New York City who has established a fund for an atomic research laboratory at this Institute as a memorial to his wife. A complete description of this equipment is soon to be published. We are glad of this first opportunity to express our heartfelt appreciation of Dr. Watters' generous support.

\section{NomenClature}

$w=$ watts input per unit area in the focal spot $c=$ heat conductivity of target material (in joules) $\sigma=$ heat capacity of target material per unit mass $\rho=$ mass density of target material

$d=$ thickness of target from face to cooling water in $\mathrm{cm}$

$0<r<1$ is the maximum value of the ratio of the duration of the heated part of the thermal cycle to the period of the entire cycle. For small focal spots it is nearly the ratio of the focal spot diameter to the circumference described by the focal spot on the target (see Fig. 1)

$n=$ the number of cycles or gyrations per second $s=$ an index of summation which takes integer values from 1 to $\infty$

$a_{\mathrm{s}}=(\pi \rho \sigma / c)^{\frac{1}{2}}(s n)^{\frac{1}{2}}$

$\theta_{s}=a_{s} d=d(\pi \rho \sigma / c)^{\frac{1}{2}}(s n)^{\frac{1}{2}}=\theta s^{\frac{1}{2}}$

$\theta=d(\pi \rho \sigma / c)^{\frac{1}{2}} n^{\frac{1}{2}}$

$\theta_{s}^{\prime}=2 \theta_{s}$

$\theta^{\prime}=2 \theta=2 d(\pi \rho \sigma / c)^{\frac{1}{2}} n^{\frac{1}{2}}$

$p_{s}=1+\sin 2 \pi s r-\cos 2 \pi s r$

$q_{s}=1-\sin 2 \pi s r-\cos 2 \pi s r$

$F_{+}\left(\theta_{s}{ }^{\prime}\right)=\left(\sinh \theta_{s}{ }^{\prime}+\sin \theta_{s}{ }^{\prime}\right) /\left(\cosh \theta_{s}{ }^{\prime}+\cos \theta_{s}{ }^{\prime}\right)$

$F_{-}\left(\theta_{s}{ }^{\prime}\right)=\left(\sinh \theta_{s}{ }^{\prime}-\sin \theta_{s}{ }^{\prime}\right) /\left(\cosh \theta_{s}{ }^{\prime}+\cos \theta_{s}{ }^{\prime}\right)$ $\alpha=$ the lowest value of $s$ for which $\theta_{s}{ }^{\prime} \geqq 2 \pi$

$\alpha=$ the lowest positive integer greater than $4 \pi^{2} / \theta^{\prime 2}$ or $\pi^{2} / \theta^{2}$

$$
x=\sum_{s=1}^{\alpha-1} \frac{1}{s \theta^{\prime}} F_{-}\left(\theta^{\prime}\right) \quad x^{\prime}=\frac{1}{\theta^{\prime}} \sum_{s=\alpha}^{\infty} s^{-1}
$$$$
y=\sum_{s=1}^{\alpha-1} \frac{1}{s \theta^{\prime}} F_{+}\left(\theta^{\prime}\right) \sin 2 \pi s r ; y^{\prime}=\frac{1}{\theta^{\prime}} \sum_{s=\alpha}^{\infty} s^{-\frac{s}{2}} \sin 2 \pi s r
$$$$
z=\sum_{s=1}^{\alpha-1} \frac{1}{s \theta^{\prime}} F_{-}\left(\theta^{\prime}\right) \cos 2 \pi s r ; z^{\prime}=\frac{1}{\theta^{\prime}} \sum_{s=\alpha}^{\infty} s^{-\frac{3}{2}} \cos 2 \pi s r
$$

$X=x+y-z+x^{\prime}+y^{\prime}-z^{\prime}$

$T^{*}=$ permissible surface temperature of target for safe operation

$T_{\max }=$ maximum temperature attained on the target surface for a mobile focal spot

$T_{0}=$ temperature of the cooling water

$T_{s}=$ temperature of a stationary focal spot

$R=\left(T_{\max }-T_{0}\right) /\left(T_{s}-T_{0}\right)=$ ratio of temperature rise in a moving spot to that in a stationary spot

$1 / R=W_{m} / W_{s}=$ multiplication of permissible watts input for a mobile focal spot with respect to a stationary focal spot

$R=r+X / \pi$. 